adopt a 'superposition' state in which they are simultaneously emitted into the surrounding environment but also localized around the emitting atoms. On page 589, Krinner et al. ${ }^{4}$ report the first observation of the dynamics of these exotic decays, not using photons, but using a system of trapped, ultracold atoms.

The atoms in Krinner and colleagues' experiments are trapped in optical lattices ${ }^{5}$, which are formed by the interference of counter-propagating laser fields - the interference generates a periodic pattern of light intensity in which the atoms are confined. Given their quantum-mechanical nature, the atoms can tunnel between neighbouring sites in the lattice at a rate that can be adjusted by altering the lasers' intensities. Because such systems are highly controllable and exhibit low decoherence (the atoms are well isolated from their environment and thus behave ideally), they are an almost perfect platform for simulating complex quantum problems found in fields such as condensed-matter and highenergy physics (see go.nature.com/2uied19). Krinner et al. now demonstrate that trappedatom systems can also be used to simulate quantum optical problems.

The authors' experiments are based on a proposal ${ }^{6}$ published in 2008 . The idea is to use atoms - in this case, rubidium atoms - that have two internal states, which respond to a one-dimensional optical lattice in different ways (Fig. 1). One state (let's call it the $f$ state) 'sees' regions of high light intensity as deep optical potential-energy wells from which it cannot move, whereas the other (the $a$ state) hardly notices the wells, so that atoms in that state can propagate through the optical lattice as a matter wave. An atom in state $f$ thus represents a matter-wave emitter in an excited state, whereas an atom in state $a$ behaves like a photon that can be emitted through spontaneous emission. To complete the analogy with atomic decay phenomena in a structured photonic environment, the two internal states are coupled to each other using external fields, so that an initial excitation can be transformed into a propagating matter wave.

In their experiments, Krinner et al. model the simplest scenario of spontaneous emission in a photonic crystal, but one that potentially offers the most insight into such processes: the spontaneous emission of a single photon into the vacuum. By tuning the experimental parameters of their system, they observed a phenomenon known as fractional decay ${ }^{5}$, in which the emitter ends up in a quantum superposition of being both excited and having decayed to the ground state. The authors also report direct evidence that the probability of the emitter remaining in the excited state does not decrease exponentially over time. Both the non-exponential behaviour and the fractional decay are some of the most peculiar effects that photonic crystals induce in quantum emitters. The new measurements are analogous to previously reported measurements of the spontaneous emission of photons in the visible-light ${ }^{7,8}$ and microwave ${ }^{9}$ regions of the electromagnetic spectrum, but in those studies, it was not possible to measure the dynamics of the decay.

The authors' experimental platform offers several useful features in addition to its excellent controllability and low decoherence. First, it is flexible enough to emulate photonic crystals that have different geometries, and to model 3D environments in which further unusual features of spontaneous emission emerge $^{10,11}$. It also allows access to parameter regimes that are out of reach of optical implementations, such as situations in which the coupling between emitters and the environment is very strong. Moreover, it might enable spontaneous emission to be studied in environments that are even more exotic than photonic crystals, such as in materials known as topological insulators - although the experimental set-up would need to be adapted so that the atoms move in the way that simulates the movement of excitations in such materials, and the efficiency with which decays can be detected would need to be improved.

Together with photonic platforms in the optical and microwave regimes, Krinner and colleagues' system opens the way to studies of the physics that emerges in unconventional quantum optical set-ups. If the experiments are extended to include many emitters, it might be possible to observe collective spontaneousemission phenomena that cannot be predicted using current computational methods, or even to engineer interactions among the emitters that cannot be produced using other platforms.

Alejandro González Tudela and J. Ignacio

Cirac are in the Theory Division of the Max

Planck Institute of Quantum Optics, Garching, D-85748 Germany.

e-mail:ignacio.cirac@mpq.mpg.de

1. Purcell, E. M. Phys. Rev. 69, 681 (1946).

2. Yablonovitch, E. Phys. Rev. Lett. 58, 2059 (1987).

3. John, S. \& Quang, T. Phys. Rev. A 50, 1764 (1994)

4. Krinner, L., Stewart, M., Pazmiño, A., Kwon, J. \& Schneble, D. Nature 559, 589-592 (2018)

5. Bloch, I., Dalibard, J. \& Zwerger, W. Rev. Mod. Phys. 80, 885 (2008).

6. De Vega, I., Porras, D. \& Cirac, J. I. Phys. Rev. Lett. 101, 260404 (2008).

7. Hood, J. D. et al. Proc. Natl Acad. Sci. USA 113, 10507 (2016).

8. Hoeppe, U. et al. Phys. Rev. Lett. 108, 043603 (2012).

9. Liu, Y. \& Houck, A. A. Nature Phys. 13, 48-52 (2017)

10.González-Tudela, A. \& Cirac, J. I. Phys. Rev. Lett. 119, 143602 (2017)

11.González-Tudela, A. \& Cirac, J. I. Phys. Rev. A 97 043831 (2018)

\title{
MICROBIOLOGY
}

\section{Viruses cooperate to defeat bacteria}

\begin{abstract}
It emerges that viruses called phages, which infect bacteria, can suppress the bacterial immune system during an initial wave of unsuccessful infection, enabling subsequent viral infection to succeed.
\end{abstract}

\section{AUDE BERNHEIM \& ROTEM SOREK}

$\mathrm{B}$ acteria and the viruses that infect them, known as phages, are engaged in a constant arms race. Bacteria continually evolve new mechanisms of resistance against viruses, while phages evolve countermeasures to overcome these defence mechanisms. Writing in Cell, Borges et al. ${ }^{1}$ and Landsberger et al. ${ }^{2}$ reveal how phages can 'collaborate' to shut down the bacterial immune system and achieve a successful infection. Although an initial viral attempt at infection fails, this enables a subsequent phage infection to be successful. This example of 'cooperation' between genetically identical individuals of a viral population illuminates a previously unknown group strategy of phages, and provides an interesting example of viral 'altruism'.

The bacterial anti-phage defence system called CRISPR-Cas recognizes and targets foreign nucleic acids in a sequence-specific manner ${ }^{3}$. To block CRISPR-Cas defences, phages express genes that encode proteins that inhibit the function of the CRISPR-Cas machinery ${ }^{4,5}$. Because these anti-CRISPR proteins are encoded in the phage genome (Fig. 1), a phage must enter a bacterial host cell and begin to express them to mount a counter-attack. Yet this raises a conundrum. CRISPR-Cas defences can attack phage DNA as soon as it enters a bacterial cell ${ }^{6}$, before the phage gets a chance to express and use its anti-CRISPR proteins. So what purpose do anti-CRISPR proteins serve if the help they provide is likely to arrive too late for the individual virus that expresses them?

To investigate this, Borges, Landsberger and their respective colleagues studied the bacterium Pseudomonas aeruginosa and examined viral anti-CRISPR proteins that target a version of the bacterial CRISPR-Cas system called type I-F. Both groups observed that the initial ratio between the number of phages and the 


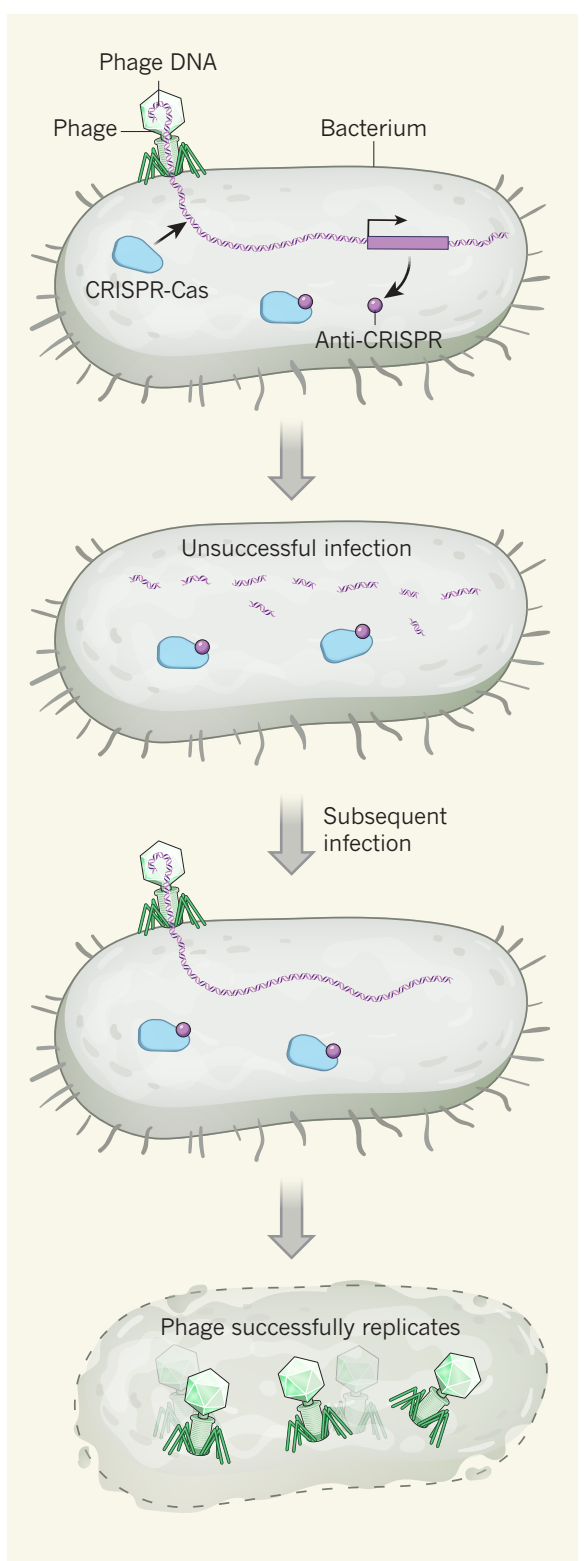

Figure 1 | A viral mechanism to thwart bacterial defences. The bacterial protein and RNA complex termed CRISPR-Cas recognizes and destroys foreign DNA, such as that of invading bacteria-infecting viruses called phages, in a sequence-specific manner ${ }^{3}$. Some phages encode anti-CRISPR proteins that bind to CRISPR-Cas and inhibit its function ${ }^{4,5}$. However, how such proteins aid viruses has been unclear, given that an initial infecting phage is probably targeted and destroyed by CRISPR-Cas, even if the phage expresses anti-CRISPR proteins before it is eliminated. Borges et al. ${ }^{1}$ and Landsberger et al. ${ }^{2}$ provide evidence that an initial unsuccessful phage infection that leads to expression of anti-CRISPR proteins generates an immunosuppressed bacterium that could then succumb to a subsequent phage infection, thereby facilitating successful phage replication.

number of bacterial host cells, known as the multiplicity of infection (MOI), could be used to predict whether phage infection would be successful. When the authors tested bacteria that lacked CRISPR-Cas immunity, even a low dose of phages resulted in successful viral propagation that caused the eventual demise of the bacterial culture. However, for bacteria and phage populations encoding CRISPRs and anti-CRISPRs, respectively, phages managed to replicate only if the initial MOI was high.

The two groups followed different strategies to determine why the initial ratio of phages to bacteria is important for the success of phage propagation. Borges and colleagues used a genetic approach to test whether the inactivation of bacterial defences was due to the presence of anti-CRISPR proteins from more than one phage in the same host cell. The authors engineered a phage that was incapable of replication but did express antiCRISPR proteins. By mixing these engineered phages with wild-type ones, the authors showed that the presence of the engineered phages could enable a wild-type phage infection to succeed even at a low MOI that would normally fail.

Landsberger and colleagues, by contrast, created a mathematical model to investigate the observed MOI dependence of phage infections. They could recapitulate their experimental findings only by using a model simulation in which bacteria enter an immunosuppressed state after surviving an initial infection by a phage that expressed anti-CRISPR proteins.

Both groups' results suggest that the first wave of phages that attempt infection succumb to CRISPR-Cas defences but manage to deliver anti-CRISPR proteins that immunosuppress the bacterial cell. This initial attack paves the way for a second wave of phages to successfully infect the now-defenceless bacterium. Having a high MOI increases the probability that a second wave of phage infection will occur.

The authors of these two studies validated this hypothesis in different ways. Landsberger and colleagues infected a bacterial population with phages encoding anti-CRISPR proteins and then introduced a type of circular DNA called a plasmid into the bacteria. Plasmid DNA would normally be targeted by the bacterial immune system, but the earlier phage infection limited this targeting of plasmid DNA, demonstrating that the bacterial cells were in an immunosuppressed state. Borges et al. demonstrated that phage-mediated immune suppression also occurs for another type of CRISPR-Cas system, called type II, suggesting that phage cooperation to enable immunosuppression of bacteria is a general principle that extends beyond a single type of CRISPR-Cas system.

The studies by Borges, Landsberger and their colleagues provide fresh insights into viral group dynamics, and join a growing body of evidence indicating that viruses can benefit from the group behaviour of a viral population. For example, phages can coordinate their infection dynamics using communication by small molecules to determine whether an individual phage will replicate in an infected cell or enter a dormant state termed lysogeny ${ }^{7,8}$. Perhaps phages also cooperate to overcome 
components of the bacterial immune system other than CRISPR-Cas, such as restriction enzymes or other antiviral defence systems ${ }^{9}$.

Many questions remain to be answered concerning the bacterial immunosuppression generated by anti-CRISPR proteins. How long will a bacterium remain in this defenceless state? How does this state vary from cell to cell in a bacterial population? Although this cooperation strategy seems to provide one way for phages to neutralize CRISPR-Cas complexes, perhaps other strategies remain to be discovered that do not require the sacrifice of the first wave of infecting phages.

Aude Bernheim and Rotem Sorek are in the Department of Molecular Genetics, Weizmann Institute of Science, Rehovot 76100, Israel. e-mail:rotem.sorek@weizmann.ac.il

1. Borges, A. L. et al. Cell https://doi.org/10.1016/ j.cell.2018.06.013 (2018).

2. Landsberger, M. et al. Cell https://doi. org/10.1016/j.cell.2018.05.058 (2018).

3. Hille, F. et al. Cell 172, 1239-1259 (2018).

4. Borges, A. L., Davidson, A. R. \& Bondy-Denomy, J. Annu. Rev. Virol. 4, 37-59 (2017).

5. Pawluk, A., Davidson, A. R. \& Maxwell, K. L. Nature Rev. Microbiol. 16, 12-17 (2018).

6. Garneau, J. E. et al. Nature 468, 67-71 (2010).

7. Ptashne, M. A Genetic Switch 3rd edn (Cold Spring Harbor Laboratory Press, 2004).

8. Erez, Z. et al. Nature 541, 488-493 (2017).

9. Doron, S. et al. Science 359, eaar4120 (2018).

R.S. declares competing financial interests. See go.nature.com/216rtqk for details.

\title{
Histidine degradation boosts cancer therapy
}

\begin{abstract}
Clinical use of the anticancer drug methotrexate can be limited by its high toxicity. It emerges that a diet rich in the amino acid histidine increases the effectiveness of methotrexate treatment and lowers toxicity in mice. SEE LETTER P.632
\end{abstract}

\section{CHRISTIAN FREZZA}

$\mathrm{M}$ ethotrexate was one of the first approved anticancer drugs, and is a cornerstone of modern chemotherapy for the treatment of certain solid tumours and blood cancers. However, this therapy must often be stopped prematurely because of its high toxicity in patients' healthy cells. On page 632, Kanarek et al. ${ }^{1}$ report that degradation of the amino acid histidine can increase the sensitivity of cancer cells to methotrexate, and the results suggest that this approach can also help to lower the side effects of methotrexate treatment in mouse models.

An enzyme cofactor termed folate is required for DNA and protein synthesis. In 1948 , it was found ${ }^{2}$ that leukaemia is particularly sensitive to drugs that block folate synthesis. Methotrexate inhibits the enzyme dihydrofolate reductase (DHFR), which synthesizes the active form of folate known as tetrahydrofolate (THF) from its precursor dihydrofolate (DHF) (Fig. 1). However, when this drug is used in the clinic, it can substantially reduce the level of folate in healthy cells - a side effect that is only partially relieved by taking folate supplements. If this toxicity reaches a level that compromises the function of healthy cells, therapy may have to be stopped prematurely. There is therefore great interest in trying to reduce the side effects of methotrexate treatment.

To investigate the processes that affect the cellular response to methotrexate, Kanarek et al. used a cell line derived from a person with leukaemia. They employed a gene-editing technique that prevents the expression of individual genes throughout the entire genome in such cells, and tested whether a decrease in the expression of any particular gene reduced the cells' sensitivity to methotrexate. One such gene that they identified encodes a protein called SLC19A1, which transports folate
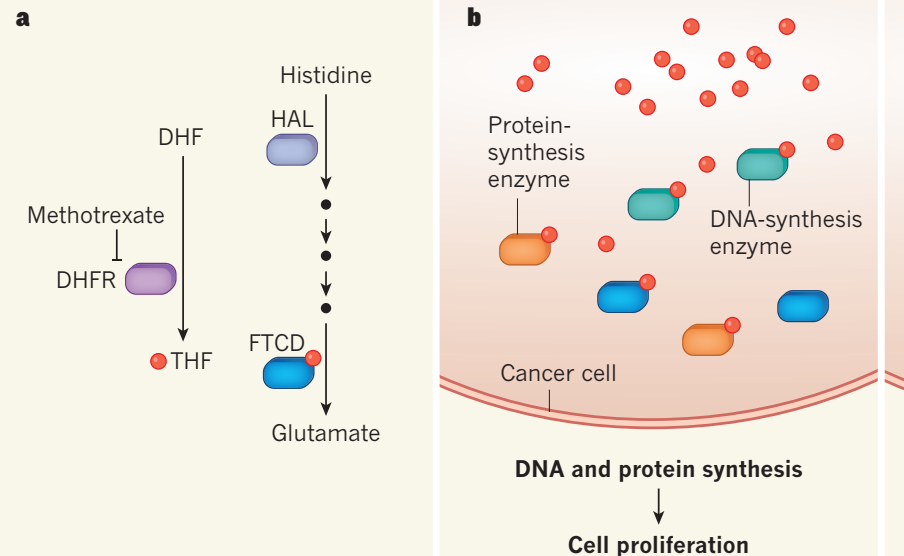

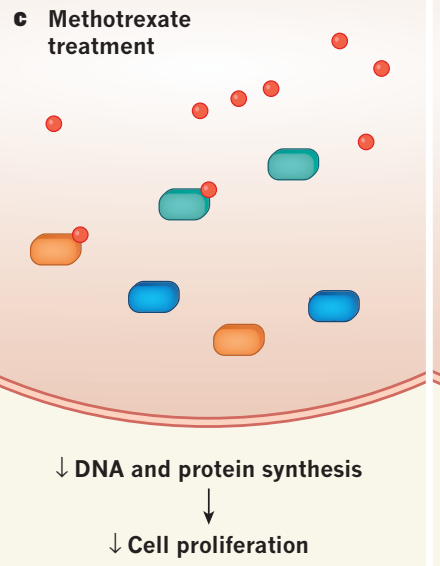

d Methotrexate + histidine treatment

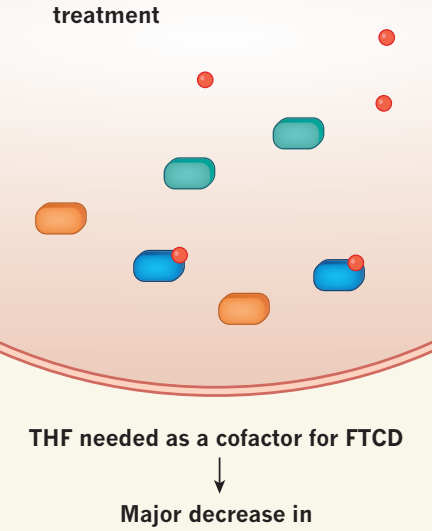

DNA and protein synthesis and in cell proliferation
Figure 1 The effectiveness of the cancer drug methotrexate can be improved by histidine degradation. a, The cofactor tetrahydrofolate (THF) is required for the function of enzymes, including those that catalyse processes needed for cell proliferation. The enzyme dihydrofolate reductase (DHFR) generates THF from dihydrofolate (DHF), and this catalytic reaction can be inhibited by the anticancer drug methotrexate. Kanarek et al. ${ }^{1}$ report that, in mouse models and human cell lines, the sensitivity of cancer cells to methotrexate can be boosted by the metabolic degradation of the amino acid histidine. Histidine is metabolized to the amino acid glutamate through a series of intermediate molecules (black circles) in a pathway that requires the enzyme histidine ammonia lyase (HAL), and, crucially for this effect on methotrexate treatment, the THF-dependent enzyme formimidoyltransferase cyclodeaminase (FTCD). b, In cancer cells, a large pool of THF is available for THF-dependent enzymes, such as FTCD or those needed for cell proliferation. c, In methotrexate-treated cancer cells, a decrease in THF levels reduces the activity of these enzymes and thereby reduces tumour growth. d, The authors report that tumour growth in mice given methotrexate and a histidine-rich diet was substantially decreased compared with $\mathbf{c}$. This might be because histidine degradation coopts THF for use with FTCD, thereby enhancing the effect of methotrexate. 